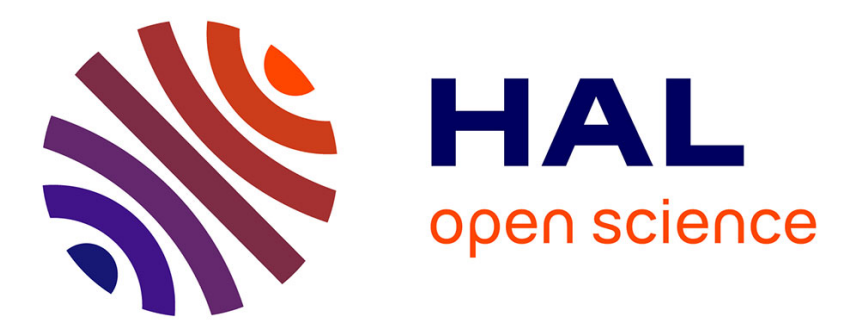

\title{
Temperature-concentration behaviour of the order parameter in the nematic phases of a lyotropic liquid crystal
}

\author{
Y. Galerne, J.P. Marcerou
}

\section{- To cite this version:}

Y. Galerne, J.P. Marcerou. Temperature-concentration behaviour of the order parameter in the nematic phases of a lyotropic liquid crystal. Journal de Physique, 1985, 46 (4), pp.589-594. 10.1051/jphys:01985004604058900 . jpa-00209999

\section{HAL Id: jpa-00209999 https://hal.science/jpa-00209999}

Submitted on 1 Jan 1985

HAL is a multi-disciplinary open access archive for the deposit and dissemination of scientific research documents, whether they are published or not. The documents may come from teaching and research institutions in France or abroad, or from public or private research centers.
L'archive ouverte pluridisciplinaire HAL, est destinée au dépôt et à la diffusion de documents scientifiques de niveau recherche, publiés ou non, émanant des établissements d'enseignement et de recherche français ou étrangers, des laboratoires publics ou privés. 


\title{
Temperature-concentration behaviour of the order parameter in the nematic phases of a lyotropic liquid crystal
}

\author{
Y. Galerne and J. P. Marcerou (*) \\ Laboratoire de Physique des Solides, Université de Paris Sud, 91405 Orsay, France
}

(Reçu le 5 septembre 1984, accepté le 10 décembre 1984)

\begin{abstract}
Résumé. - Les variations en fonction de la température des invariants du paramètre d'ordre sont mesurées dans les phases nématiques uniaxes et biaxes de différents mélanges de laurate de potassium, 1-decanol, $\mathrm{D}_{2} \mathrm{O}$. Elles montrent que les transitions de phase nématique uniaxe-biaxe sont du second ordre et de champ moyen, y compris dans le cas de la concentration réentrante qui donne la séquence nématique discotique-biaxe-discotique en température croissante.
\end{abstract}

\begin{abstract}
The temperature variations of the order parameter invariants are measured in the uniaxial and biaxial nematic phases of different mixtures of potassium laurate, 1-decanol, $\mathrm{D}_{2} \mathrm{O}$. They show that the uniaxial to biaxial nematic phase transitions are second-order and mean-field, including in the case of the reentrant concentration, which gives the sequence discotic-biaxial-discotic of nematic phases when increasing temperature.
\end{abstract}

Mixtures in aqueous solutions, of amphiphilic molecules having a hydrophilic head and a hydrophobic tail may fôrm anisotropic micellar aggregates [1] which under particular conditions, may exhibit an orientational long-range ordering characteristic of the nematic phases. According to symmetry arguments, three types of lyotropic nematic phases are possible, and indeed, have been observed : two are uniaxial [2] and another one is biaxial [3]. The two uniaxial nematic phases are known as $\operatorname{discotic}\left(\mathrm{N}_{\mathrm{L}}\right)$, and cylindric $\left(\mathrm{N}_{\mathrm{C}}\right)$, depending on their magnetic anisotropy [4]. These uniaxial phases have been studied in detail using different physical means, such as optical observations of the textures under polarizing microscope, X-ray and neutron scattering, NMRmeasurements, etc. (see for example Ref. [5] and references therein). On the contrary, the first observation of the biaxial nematic phase $\left(\mathrm{N}_{\mathrm{BX}}\right)$ is recent [3], and little information is available on it to-date [6]. In the temperature-concentration diagram (Fig. 1 of Ref. [3]), the biaxial phase appears to be an intermediate phase all along the border between the two uniaxial phases [7], as predicted by statistical models [8-10]. This phase diagram shows also that for a particular choice of temperature and concentration, reentrant

$\left(^{*}\right)$ Permanent address : Centre Paul Pascal, Domaine Universitaire, 33405 Talence, France. configurations exist; i.e. on heating the sample, the following sequence can be observed : $N_{L} \rightarrow N_{B X} \rightarrow$ $\mathrm{N}_{\mathrm{C}} \rightarrow \mathrm{N}_{\mathrm{BX}} \rightarrow \mathrm{N}_{\mathrm{L}}$.

In this paper we present optical measurements of the tensorial order parameter in the three nematic phases encountered when varying temperature in samples of different concentrations, chosen around the reentrant zone. This is a simple and convenient method to investigate the biaxial domain and its transitions to the uniaxial phases, and also to study the uniaxial phases and their transitions to the isotropic phase.

\section{Theoretical.}

1.1 ORDER PARAMETER. - The order parameter of the uniaxial and biaxial nematic phases is a second rank tensor to which the anisotropic part of the macroscopical quantities, obeying linear physics, is proportional. Because of this required property of linearity the magnetic susceptibility is usually preferred as an order parameter to the dielectric or optical susceptibility [11]. However, although the anisotropic part of the optical susceptibility is not a good order parameter in the thermotropic liquid crystals, experimentally it seems not to be bad for the lyotropics [6]. This observation can be justified by the very low birefringence value $\left(\sim 2 \times 10^{-3}\right.$, i.e. about 100 times smaller than in thermotropics), which indicates that the mutual interactions between the induced electric 
dipoles, due to the anisotropic part of the microscopical susceptibility, are negligible in the lyotropics, and therefore that the dielectric susceptibility is as good as the magnetic susceptibility for the measurement of the order parameter.

The anisotropic part of the optical susceptibility $\overline{\bar{\varepsilon}}_{\mathrm{a}}$ is expressed as a function of the optical index differences. In the eigen axes frame, referred to by the subscripts $1,2,3$, it reduces to the diagonal elements :

$$
\begin{aligned}
& \varepsilon_{\mathrm{a}_{1}}=-\frac{4\langle n\rangle}{3}\left(\left(n_{2}-n_{1}\right)+\frac{\left(n_{3}-n_{2}\right)}{2}\right) \\
& \varepsilon_{\mathrm{a}_{2}}=\frac{2\langle n\rangle}{3}\left(\left(n_{2}-n_{1}\right)-\left(n_{3}-n_{2}\right)\right) \\
& \varepsilon_{\mathrm{a}_{3}}=\frac{4\langle n\rangle}{3}\left(\frac{\left(n_{2}-n_{1}\right)}{2}+\left(n_{3}-n_{2}\right)\right)
\end{aligned}
$$

where $\langle n\rangle$ is the average index of refraction $\sim 1.375$. In the uniaxial nematic phases, these diagonal elements are proportional to the usual « orientational order parameter " $S=1 / 2\left\langle 3 \cos ^{2} \theta-1\right\rangle, \theta$ being the angle between a randomly chosen molecular axis and the symmetry axis of the nematic phase (director). In the $\mathrm{N}_{\mathrm{L}}$ phase, constituted of " disk-like " micelles, the molecules are preferably parallel to the director, while they are mostly perpendicular to it in the $\mathrm{N}_{\mathbf{C}}$ phase, due to the "cylindrical shape " of the micelles. As a consequence, the limit for a fully ordered phase is $S=1$ in the $N_{L}$ phase, and $S=-1 / 2$ in the $N_{C}$ phase.

For our study, which considers only uniformly oriented samples, no reference frame is needed. It is then very convenient to reduce the tensorial orderparameter to its symmetric invariants [12]. They are :

$$
\begin{aligned}
& \sigma_{1}=\varepsilon_{\mathrm{a}_{1}}+\varepsilon_{\mathrm{a}_{2}}+\varepsilon_{\mathrm{a}_{3}}=0 \\
& \sigma_{2}=\frac{2}{3}\left(\varepsilon_{\mathrm{a}_{1}}^{2}+\varepsilon_{\mathrm{a}_{2}}^{2}+\varepsilon_{\mathrm{a}_{3}}^{2}\right) \\
& \sigma_{3}=4 \varepsilon_{\mathrm{a}_{1}} \varepsilon_{\mathrm{a}_{2}} \varepsilon_{\mathrm{a}_{3}}
\end{aligned}
$$

$\sigma_{1}=0, \overline{\bar{\varepsilon}}_{\mathrm{a}}$ being a traceless tensor. In the uniaxial nematic phases, because the diagonal elements (1) are proportional to $S, \sigma_{2}$ and $\sigma_{3}$ are related by : $\sigma_{3}=$ $\pm \sigma_{2}^{3 / 2}$ (the sign being that of $S$, positive in $\mathrm{N}_{\mathrm{L}}$ and negative in $N_{C}$ [13]). In the biaxial phase, $\sigma_{2}$ and $\sigma_{3}$ are independent. The relation then transforms to the inequalities $-\sigma_{2}^{3 / 2}<\sigma_{3}<\sigma_{2}^{3 / 2}$ which expresses that the $\mathrm{N}_{\mathrm{BX}}$ phase is intermediate between the uniaxial $\mathrm{N}_{\mathrm{C}}$ and $\mathrm{N}_{\mathrm{L}}$ phases.

1.2 FREE ENERGY EXPANSION IN THE MEAN-FIELD THEORY. - In the mean-field theory, where the effects of fluctuations are neglected, the free energy is expanded in terms of the order parameter. The gradient terms being suppressed, all references to the laboratory frame are useless. The order parameter can therefore be reduced to the invariants, and the free energy is expanded as :

$$
G=a \sigma_{2}+b \sigma_{3}+\frac{1}{2} c \sigma_{2}^{2}+d \sigma_{2} \sigma_{3}+\frac{1}{2} e \sigma_{3}^{2},
$$

the coefficients being regular functions of temperature and concentrations. In a first order approximation, $a$ and $b$ are supposed to vary linearly with temperature while the other coefficients are constant. The minimum of $G$ is given by :

$$
\sigma_{2}=\frac{b d-a e}{c e-d^{2}}, \quad \sigma_{3}=\frac{a d-b c}{c e-d^{2}} .
$$

The values of the invariants are effective if they are physically accessible, i.e. if the condition $\sigma_{3}^{2}<\sigma_{2}^{3}$ is fulfilled; the phase is then biaxial. If this inequality is not fulfilled, the physical values of the invariants are given by the relative minimum of $G$ satisfying the constraint $\sigma_{3}= \pm \sigma_{2}^{3 / 2}$, and the phase is either uniaxial-nematic or isotropic. In this case, it is preferable to express $\sigma_{2}$ and $\sigma_{3}$ as functions of $S$. To a multiplicative constant and to the lowest orders in $S$, equation (3) becomes then the usual free-energy expansion [11] :

$$
G_{1}=a S^{2}+b S^{3}+\frac{c}{2} S^{4}
$$

This equation shows that the coefficient $a$ controls the isotropic to uniaxial-nematic phase-transition, the nature of the nematic phase being determined by the sign of $b$. Similarly, the variations of $b$, through $\sigma_{3}$ in equation (3), determine the existence of the biaxialnematic phase. This is clear in the weak-coupling limit $\left(d \simeq 0\right.$ ), where $\sigma_{3}$ is directly proportional to $b$. So, driven by the variations of $b, \sigma_{3}$ varies freely in the $\mathrm{N}_{\mathbf{B X}}$ phase until the uniaxial constraint $\left(\sigma_{3}^{2}=\sigma_{2}^{3}\right)$ is met in a continuous way, making a second-order uniaxial to biaxial nematic phase transition [12].

The uniaxial-nematic to isotropic phase-transition is, in general, first-order because of the $S^{3}$ term in $G_{1}$ [11]. It becomes weakly first-order as predicted by Shih and Alben [8] if a $N_{B X}$ phase exists in the vicinity. This point can be understood in the following manner. The jump $\Delta S$ of the order-parameter at the uniaxialnematic to isotropic transition is given by equation(5) : $\Delta S=-\frac{3 b}{4 c}$. Because the coefficient $b$ goes to zero in the biaxial phase (or close to the $\mathbf{N}_{\mathbf{B X}}$ phase in the case $d \neq 0$ ), the ratio $b / c$ may be assumed to still remain small at the nematic-isotropic transition. So, the weak value of $\Delta S$ can be understood as a consequence of the existence of the $\mathrm{N}_{\mathrm{BX}}$ phase [14].

\section{Experimental.}

2.1 SAMPLES, EXPERIMENTAL SET-UP AND METHOD. The measurements reported in the paper are performed on mixtures of potassium laurate (KL) (synthetized and recrystallized in the laboratory), 1-decanol and 
$\mathrm{D}_{2} \mathrm{O}$, of concentrations approximately chosen from the phase diagram studied by $\mathrm{Yu}$ and Saupe $[3,7]$. Three samples of increasing KL-concentration are presented here :

a) $\mathrm{KL} 25.5 /$ Decanol 6.38/ $\mathrm{D}_{2} \mathrm{O} 68.12 \mathrm{wt} \%$

b) $\mathrm{KL} 26.0 /$ Decanol $6.24 / \mathrm{D}_{2} \mathrm{O} 67.76 \mathrm{wt} \%$ (same as in Ref. [6]),

$$
\text { c) } \mathrm{KL} 26.03 / \text { Decanol } 6.24 / \mathrm{D}_{2} \mathrm{O} \quad 67.73 \mathrm{wt} \% \text {. }
$$

The samples are prepared following the usual method [3]. They are enclosed in a Hellma cell of 1 or $2.5 \mathrm{~mm}$ thickness, and hermetically sealed to prevent concentration drifts. In this way, the main physical properties are retained for weeks. The temperature of the sample is controlled by means of a thermostat (of $0.02^{\circ} \mathrm{C}$ accuracy) composed of two independent systems : a water-circulating first-stage (Haake $G / D_{3}$ ) and an electronically servo-regulated second-stage. The orientation of the sample is realized by combining a silane-treatment on the surfaces of the cell [15] with the bulk coupling of the nematic director to a magnetic field $\mathbf{H}(\sim 16 \mathrm{kG})$. For this reason, all the experimental set-up is built between the poles of an electromagnet. In this manner, the sample can be oriented correctly enough for measuring the refractive indices by conoscopy, its director being aligned parallel to $\mathbf{H}$ in the $\mathrm{N}_{\mathrm{C}}$ phase, and homeotropic (i.e. perpendicular to the surfaces of the cell) in the $\mathrm{N}_{\mathrm{L}}$ phase. In this latter case, the orientation is achieved by repeated rotations of the sample around the normal to the cell in the presence of $\mathbf{H}$. These two axes, the (horizontal) magnetic field and the (vertical) rotational axis, define the laboratory frame. They are respectively, the 1 axis and 3 axis along which the director aligns in both uniaxial-nematic phases (Fig. 1). The measurement of the optical indices is done using a conoscopic method which is realized with a He-Ne laser beam converging in the sample by means of a wide-aperture microscopeobjective (half-angle of the aperture $\sim 50 \mathrm{deg}$.). In this manner, a very extensive interference pattern is obtained, allowing for accurate measurements of optical index differences (to about $10^{-5}$ ).

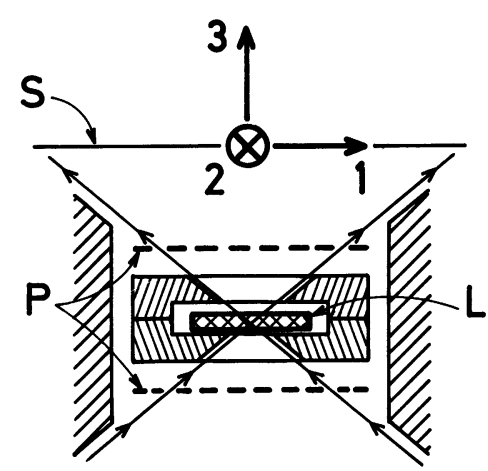

Fig. 1. - Schematic set up. The sample $L$ is placed inside a thermostat, between the poles of an electromagnet. It is illuminated by a strongly converging laser beam. The light, polarized before and after the sample (with the polarizers $\mathrm{P}$ ), interferes on the screen $S$ parallel to the 1-2 plane.
A series of photographs of these interferences is shown in figure 2 (except Fig. $2 \mathrm{f}$ which gives the directions of the polarizers and of the axes of the laboratory frame). Figure $2 \mathrm{a}$ is the typical conoscopic pattern of the discotic-uniaxial phase homeotropically aligned along the 3 axis; figures $2 b, c, d$ are photos of the biaxial nematic phase with the eigen directions along the axes of the laboratory frame; and figure $2 \mathrm{e}$ shows the pattern obtained in the $\mathrm{N}_{\mathrm{C}}$ phase oriented along the 1 axis. Note that no qualitative change occurs between figure $2 \mathrm{~d}$ and figure $2 \mathrm{e}$, and that a simple observation of the conoscopic pattern is hardly able to determine the temperature of the $\mathrm{N}_{\mathbf{B X}}$ to $\mathrm{N}_{\mathrm{C}}$ phase transition. The positions of the interference fringes are measured at equilibrium along the 1 and 2 axes, for the two orientations of the polarizers, perpendicular and parallel, which correspond respectively to measurements of integer and half integer interference orders. These values, together with the sample thickness and the average index $\langle n\rangle \sim 1.37$, are introduced in a least squares fit. Both the index differences $n_{2}-n_{1}$ and $n_{3}-n_{2}$ are thus found. This method does not give the sign of $n_{2}-n_{1}$ and $n_{3}-n_{2}$ which is found independently by looking at the modification of the conoscopic pattern when a slide of known-birefringence material is superposed on the sample.

\subsection{Results.}

2.2.1 Index differences versus temperature. - Typical measurements of the index differences $n_{2}-n_{1}$ and $n_{3}-n_{2}$ are shown in figure 3 as functions of temperature, the letters $a, b, c$, indicating the concentrations

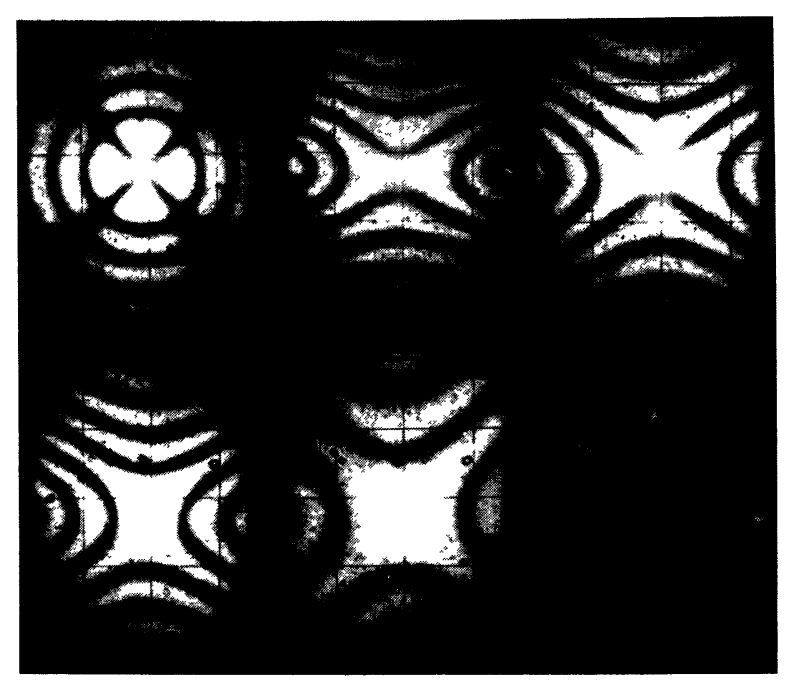

Fig. 2. - Conoscopic patterns of the $2.5 \mathrm{~mm}$-thick sample $b$ : a) $\mathrm{N}_{\mathrm{L}}$ phase in the homeotropic orientation (director along the 3 axis). b), c) and d) $N_{B x}$ phase when increasing temperature from the $N_{L}$ to the $N_{C}$ transition. e) $N_{C}$ phase in the planar orientation (director along the 1 axis). $f$ ) Laboratory-frame axes and polarizers $P$; the 1 axis is parallel to the magnetic field and the 3 axis is perpendicular to the plates of the sample. 

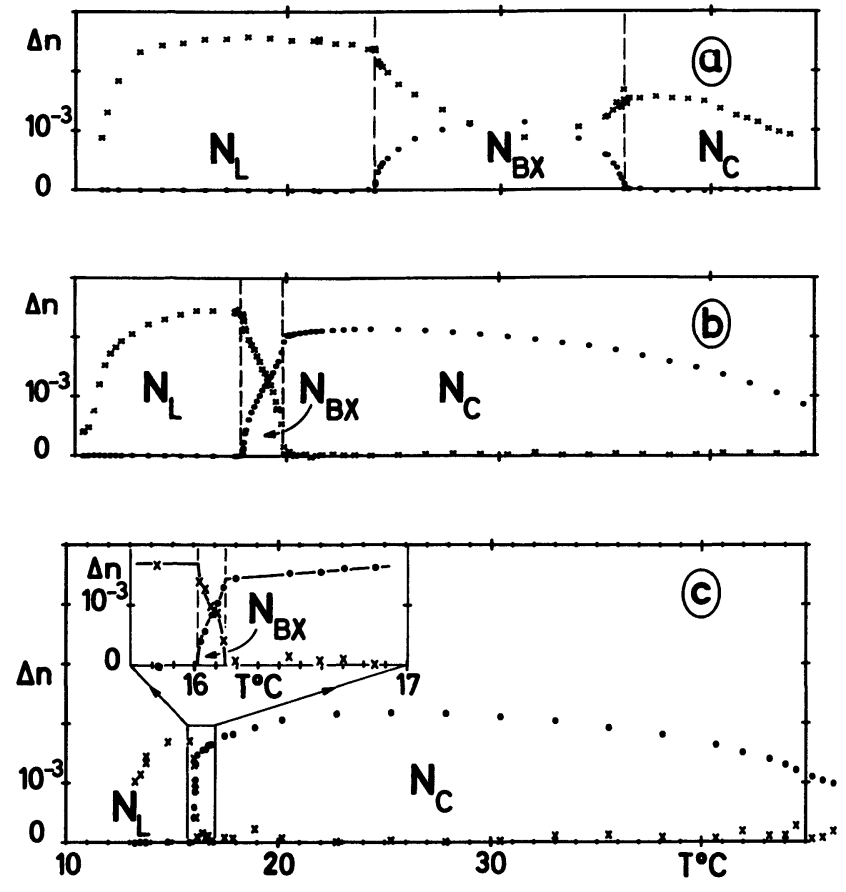

Fig. 3. - Index differences $\left(n_{2}-n_{1}: \bullet ; n_{3}-n_{2}: \times\right)$ measured as functions of temperature in the samples $a, b$, and $c$ respectively. Because they are thinner $(1 \mathrm{~mm})$ the samples $a$ and $c$ give less accurate measurements than sample $b$ $(2.5 \mathrm{~mm})$. Sample $c$ has a narrow $\mathrm{N}_{\mathrm{BX}}$ temperature range. The insert displays an expansion by a factor 10 of the temperature scales. The full line in the insert is just a guide for the eye.

of the different samples. The uniaxial phases, and their transitions to the biaxial phase, appear clearly in these plots. They correspond to one vanishing index difference : $n_{2}-n_{1}=0$ in $\mathrm{N}_{\mathrm{L}}, n_{3}-n_{2}=0$ in $\mathrm{N}_{\mathrm{C}}$. In samples $b$ and $c$ (Figs. $3 b$ and 3c), the succession $\mathbf{N}_{\mathbf{L}}-\mathbf{N}_{\mathbf{B X}}-\mathbf{N}_{\mathbf{C}}$ is thus encountered when increasing temperature. In sample $a$ which contains less $\mathrm{KL}$ than the two other samples, the reentrant sequence $N_{L}-N_{B X}-N_{L}$ is observed. In samples of lower KL-concentration, only the $N_{L}$ phase is found [3,7].

From these results, one can readily notice some general features.

In every cases, when both the $\mathrm{N}_{\mathrm{L}}$ and $\mathrm{N}_{C}$ phases exist, the $\mathrm{N}_{\mathrm{BX}}$ phase appears as an intermediate phase which prevents a direct $\mathrm{N}_{\mathrm{L}}-\mathrm{N}_{\mathrm{C}}$ transition (Figs. 3b, 3c). As is discussed in $\S 2.2 .2$, this point is consistent with the mean-field analysis.

From the index differences, $n_{3}-n_{2}$ in $\mathrm{N}_{\mathrm{L}}$ and $n_{2}-n_{1}$ in $\mathrm{N}_{\mathrm{C}}$, one can deduce an estimate of the temperature variations of the order parameter $S$ in the uniaxial phases. Because the maximum index difference in $\mathrm{N}_{\mathrm{L}}$ is smaller than twice the maximum index difference in $N_{C}$, it is suggested that the $N_{L}$ phase has, in general, a lower order parameter than the $\mathrm{N}_{C}$ phase. This point is, to a certain extent, confirmed by X-ray measurements [16].
No hysteresis to within our $0.02{ }^{\circ} \mathrm{C}$ temperature resolution is observed at the uniaxial to biaxial phase transitions, nor is any jump of the index differences measured. This confirms that the uniaxial to biaxial phase transitions are second-order [17] as predicted by the mean-field theories.

On the contrary, the isotropic to $\mathrm{N}_{\mathrm{L}}$ phase transition is weakly first-order as is shown by the small jump of the birefringence data (at $T=11^{\circ} \mathrm{C}$, Fig. 3b), though partly rounded off in the coexistence region. The $\mathbf{N}_{C}$ to isotropic phase change is also measured to be weakly first-order, for the same reasons, but with less accuracy because of the planar orientation of the $\mathrm{N}_{\mathrm{C}}$ phase. The order parameter jump in both nematicisotropic transitions can be estimated to $\sim 0.2$ if one supposes that the maximum of $S$, around the biaxial phase is about 0.8 [16]. The same estimate performed on a sample not having a biaxial phase (sample of lower KL-concentration than sample $a$ ) gives $\Delta S \sim 0.4$ at the $\mathrm{N}_{\mathrm{L}}$-isotropic transitions which indicates a usual first-order nematic-isotropic transition. This is a confirmation of the predictive criterion of Shih and Alben [8] that the existence of an $\mathbf{N}_{\mathbf{B X}}$ phase is connected to a weakly first-order nematic to isotropic phase transition.

2.2.2 Invariants of the order parameter. - As previously discussed $(\S 1.1)$ it is convenient to represent the experimental data using the invariants of the order parameter. Figure 4 displays the invariants $\sigma_{2}$ and $\sigma_{3}$ as functions of temperature in the biaxial domain of sample $b$. It shows that $\sigma_{2}$ and $\sigma_{3}$ behave linearly with temperature, within the experimental uncertainties, according to mean-field theory. Figure 5 represents a similar result in the narrow temperature range of the $\mathrm{N}_{\mathrm{BX}}$ phase of sample $c$. Note that this measurement is rather a demonstration of a practical method for determining the uniaxial to biaxial phase transitions by just drawing the linear intercepts in $\sigma_{3}(T)$.

In fact, the linear variations of $\sigma_{2}(T)$ and $\sigma_{3}(T)$ in the $\mathrm{N}_{\mathrm{BX}}$ phase are only valid as a first-order approximation. It concerns narrow biaxial temperatureranges only $\left(\sim 1^{\circ} \mathrm{C}\right.$ if compared to our accuracy of measurement). Around the reentrant zone where a wide biaxial domain $\left(\sim 10^{\circ} \mathrm{C}\right)$ exists, the linear approximation has to be rejected. Moreover, reentrance being typically a return to the initial $\mathrm{N}_{\mathrm{L}}$ phase, linear behaviours for $\sigma_{2}(T)$ and $\sigma_{3}(T)$ are clearly impossible. Figure 6 shows such a non-linear behaviour of $\sigma_{3}(T)$, as measured in sample $a$. The linear approximations remain, however, possible over reduced (but finite) temperature ranges $\left(\sim 1^{\circ} \mathrm{C}\right)$, even near the uniaxial-biaxial transitions. This indicates that the uniaxial-biaxial transitions are mean-field also in this case. If they were critical, the invariants would vary like $\left|T-T_{\mathrm{c}}\right|^{2 \beta}$ near the transitions $\left(T_{\mathrm{c}}\right.$ : transition temperature, $\beta \sim 1 / 3[18]$ ), which would be marked by infinite derivatives $\mathrm{d} \sigma / \mathrm{d} T$ on the biaxial side of the uniaxial-biaxial transitions. Such a critical behaviour 

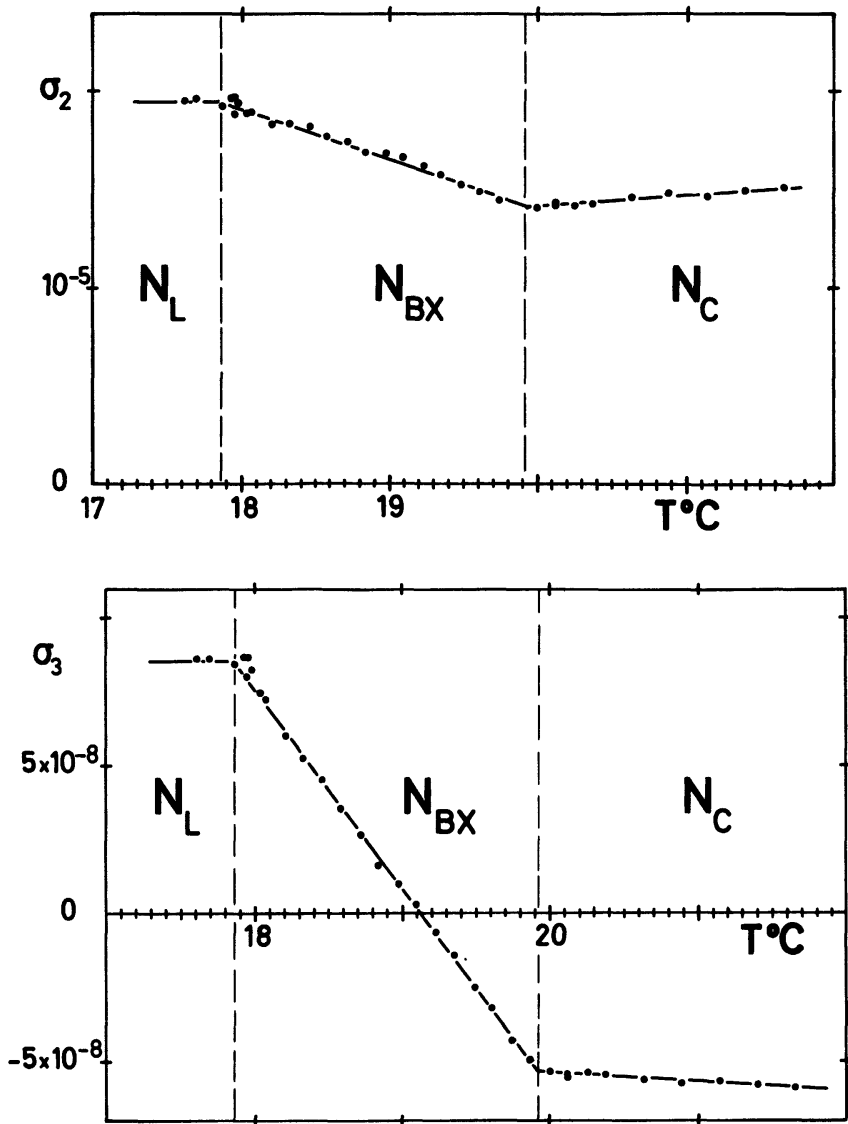

Fig. 4. - Symmetric invariants versus temperature in the $\mathrm{N}_{\mathrm{Bx}}$ phase of sample $b: \sigma_{2}(T)$ and $\sigma_{3}(T)$, respectively up and down.

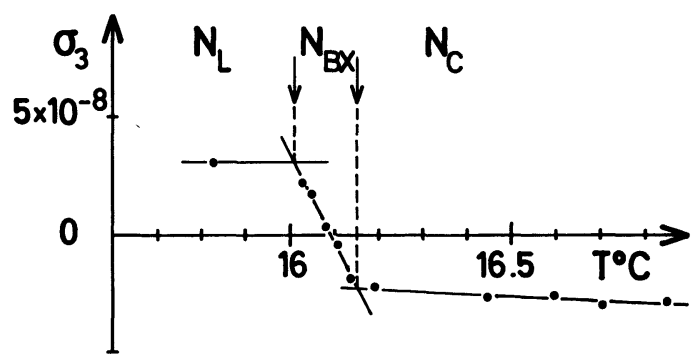

Fig. 5. $-\sigma_{3}(T)$ in the $\mathrm{N}_{\mathrm{BX}}$ phase of sample $c$. Such a plot yields a practical way for determining the uniaxial to biaxial nematic transitions.

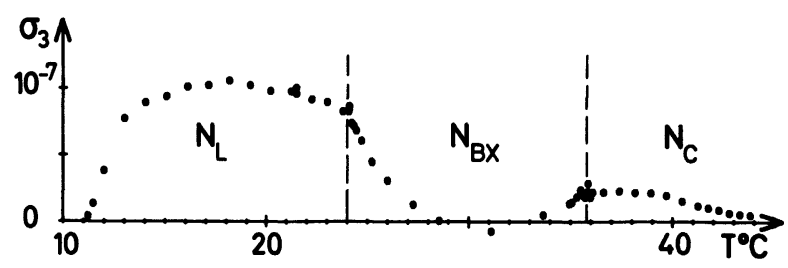

Fig. 6. $-\sigma_{3}(T)$ in the reentrant sequence $\mathrm{N}_{\mathrm{L}}-\mathrm{N}_{\mathrm{BX}}-\mathrm{N}_{\mathrm{L}}$ of sample $a$. The linear approximation is no longer possible on the whole $\mathbf{N}_{\mathbf{B X}}$ range, but remains valid on smaller scales $\left(\sim 1^{\circ} \mathrm{C}\right)$, especially near the uniaxial-biaxial transitions, which are therefore mean-field. is not observed within the accuracy of our $T$ and $\sigma$ measurements [19].

The results of the order parameter measurements can advantageously be collected in a $\left(\sigma_{2}, \sigma_{3}\right)$-plot (Figs. 7a and $\mathrm{b}$ according to the sample). The arrows indicate the variations of the point $\left(\sigma_{2}, \sigma_{3}\right)$ as the temperature is increased. In the uniaxial phases, it follows the branches which correspond to the constraint $\sigma_{3}= \pm \sigma_{2}^{3,2}\left(\operatorname{sign}+\right.$ for $\mathrm{N}_{\mathrm{L}},-$ for $\mathrm{N}_{\mathrm{C}}$ ). Its position $\left(\sigma_{2}, \sigma_{3}\right)$ is given by the minimum of the free energy $G$ in the physically accessible $\sigma$-space. If we make the simple assumption that $G\left(\sigma_{2}, \sigma_{3}\right)$ is a oneminimum function continuously varying with temperature, it results in continuous.variations for $\left(\sigma_{2}, \sigma_{3}\right)$ with temperature, which implies the impossibility of going directly from the $\mathrm{N}_{\mathrm{L}}$ to the $\mathrm{N}_{\mathrm{C}}$ phase without crossing the $\mathrm{N}_{\mathrm{BX}}$ phase. Such a behaviour, which has been predicted by statistical models [8-10], is consistent with all the phase diagrams $[3,7]$ in which the $N_{L}$ and $N_{C}$ phases are both present.

\section{Conclusion.}

The measurements of the invariants of the order parameter as functions of temperature in samples
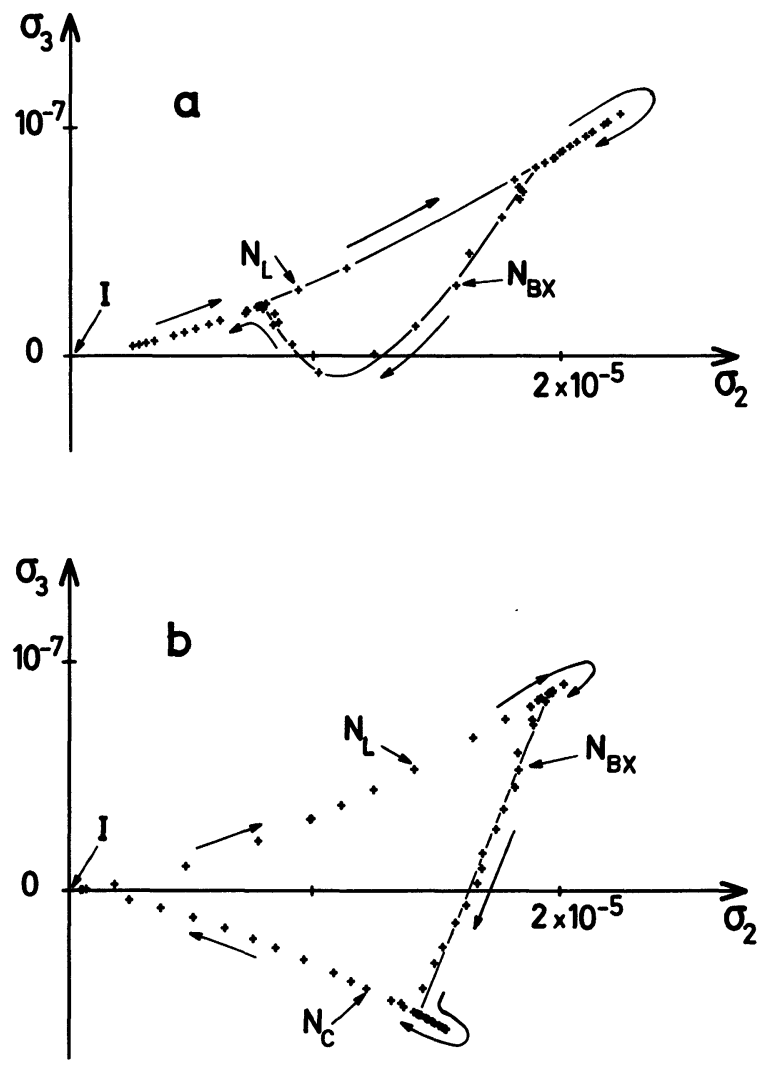

Fig. 7. $-\left(\sigma_{2}, \sigma_{3}\right)$-plots for the samples $a$ and $b$, respectively. The arrows indicate the variation of $\left(\sigma_{2}, \sigma_{3}\right)$ for increasing temperature from and back to the isotropic phase (I). The straight line in figure $b$ is a consequence of both the linear variations of $\sigma_{2}$ and $\sigma_{3}$ with temperature. 
of different concentrations, show that the uniaxial to biaxial nematic phase transitions are second-order and mean-field, to within our accuracy of measurement. This conclusion of a mean-field behaviour of the uniaxial-biaxial transitions is also valid at the reentrant concentration, though the invariants $\sigma_{2}$ and $\sigma_{3}$ then have non-linear variations with temperature. On the contrary, the uniaxial to isotropic phase transitions, which are known to be mean-field, are found to be weakly first-order when a biaxial phase exists in the vicinity, following the prediction of Shih and Alben.

\section{Acknowledgments.}

We are greatly indebted to Dr. P. Keller for preparation of the potassium laurate and to Drs. A. Figueiredo Neto and L. Liébert for their kind assistance in this work.

\section{References}

[1] Lawson, K. D. and Flautt, T. J., J. Am. Chem. Soc. 89 (1967) 5489.

[2] Radley, K., Reeves, L. W. and Tracey, A. S., J. Phys. Chem. 80 (1976) 174.

[3] Yu, L. J. and Saupe, A., Phys. Rev. Lett. 45 (1980) 1000. Saupe, A., Boonbrahm, P., Yu, L. J., J. Chimie Phys. 80 (1983) 7.

[4] Charvolin, J., Levelut, A. M., Samulski, E. T., J. Physique Lett. 40 (1979) L-587.

[5] Hendrikx, Y., Charvolin, J., Rawiso, M., Liébert, L., Holmès, M. C., J. Phys. Chem. 87 (1983) 3991.

[6] Galerne, Y., Marcerou, J. P., Phys. Rev. Lett. 51 (1983) 2109.

[7] Figueiredo Neto, A. M., Liébert, L., Galerne, Y., to be published.

[8] Shin, C. S., Alben, R., J. Chem. Phys. 57 (1972) 3055. Alben, R., Phys. Rev. Lett. 30 (1973) 778.

Alben, R., J. Chem. Phys. 59 (1973) 4299.

[9] Rabin, Y., McMullen, W. E., Gelbart, W. M., Mol. Cryst. Liq. Cryst. 89 (1982) 67.

[10] Chen, Z. Y. and Deutch, J., J. Chem. Phys. 80 (1984) 2151 .

Caflish, R., Chen, Z.-Y., Berker, A., Deutch, J., Phys. Rev. A 30 (1984) 2562.

[11] DE Gennes, P. G., The Physics of Liquid Crystals (Clarendon Press, Oxford) 1974.

[12] Freiser, M. J., Phys. Rev. Lett. 24 (1970) 1041.

[13] One can equivalently notice that the uniaxial condition may be written $\varepsilon_{\mathrm{a}_{1}}=\varepsilon_{\mathrm{a}_{2}}<0$ in the $\mathrm{N}_{\mathrm{L}}$ phase, and $\varepsilon_{\mathrm{a}_{2}}=\varepsilon_{\mathrm{a}_{3}}>0$ in the $\mathrm{N}_{\mathrm{C}}$ phase which implies $\sigma_{3}=\sigma_{2}^{3 / 2}$, and $\sigma_{3}=-\sigma_{2}^{3 / 2}$, respectively.

[14] Note inversely that lyotropics can have weakly first- order uniaxial-nematic to isotropic phase transitions without any evidence of a neighbouring $\mathrm{N}_{\mathrm{BX}}$ phase ; see : Rosenblatt, C., Kumar, S., Litster, J. D., Phys. Rev. A 29 (1984) 1010.

[15] Bartolino, R., Chiaranza, T., Meuti, M., CompaGNONI, R., Phys. Rev. A 26 (1982) 1116.

[16] Figueiredo Neto, A. M., Galerne, Y., Levelut, A. M., LIÉBERT, L., to be published.

[17] The possibility of a first order transition smoothed by a coexistence region between the $\mathrm{N}_{L}$ and $\mathrm{N}_{C}$ phases must also be discarded though such a demixtion would optically very much resemble a biaxial phase. Two arguments may be proposed. First, the biaxial samples remain clear for months, indicating that the demixtion, if it exists, did not reach the $1 \mu \mathrm{m}$-scale after this period. Second, the measured temperature variations of the index differences, which display infinite derivatives at each uniaxial-biaxial phase transition, are not consistent with the smoothing effects that a demixtion would produce. For these reasons, the uniaxial to biaxial nematic-phases transitions observed in our lyotropic systems, are secondorder phase transitions, and the $\mathrm{N}_{\mathrm{Bx}}$ phase is really a phase and not a demixtion.

[18] Boonbrahm, P. and Saupe, A., J. Chem. Phys. 81 (1984) 2076.

[19] Note that the uniaxial-nematic to isotropic phase transitions are known to be mean-field, though first order, in lyotropics as in thermotropics; see Kumar, S., Yu, L. J., Litster, J. D., Phys. Rev. Lett. 50 (1983) 1672. 